\title{
PILOT STUDY OF GAZE SCANNING AND INTERSECTION DETECTION FAILURES BY DRIVERS WITH HEMIANOPIA
}

\author{
Alex R. Bowers, Concetta F. Alberti, Alex D. Hwang, Robert Goldstein and Eli Peli \\ Schepens Eye Research Institute, Harvard Medical School \\ Boston, MA \\ alex_bowers@meei.harvard.edu
}

\begin{abstract}
Summary: In a prior study, intersection detection failures of individuals with hemianopia were strongly associated with inadequate head scanning; however, eye position was not tracked. In this pilot study, we tracked eye and head movements, and examined the relationship between gaze scanning and detection of pedestrians at intersections in a driving simulator. Gaze scan deficits, in particular not scanning sufficiently far into the blind hemifield, were the main reason for detection failures at the extreme edge of the clear-sight triangle in the blind hemifield. In addition, the gaze data revealed detection failures due to looked-but-failed-to-see events. The results suggest that $\mathrm{HH}$ drivers may be at increased risk for collisions at intersections.
\end{abstract}

\section{BACKGROUND AND OBJECTIVES}

Homonymous hemianopia $(\mathrm{HH})$ is the loss of half of the visual field on the same side in both eyes that commonly occurs following stroke and traumatic brain injury. Twenty-two states prohibit people with $\mathrm{HH}$ from driving as they do not meet the minimum field extent requirement (e.g. $120^{\circ}$ in Massachusetts). However, in other states they may be permitted to drive, and in some countries (e.g., Belgium, Netherlands, Switzerland, UK, Canada) they may be licensed after taking a specialized road test. Individuals with $\mathrm{HH}$ may be able to compensate for their hemifield loss by scanning with eye and/or head movements into the blind field; however, there is only limited information about their scanning behaviors when driving (Bowers et al., 2014; Papageorgiou et al., 2012; Wood et al., 2011). In an on-road study of drivers with HH (Bowers et al., 2012), the majority of detection failures occurred at intersections, suggesting that many of the participants did not scan effectively; however, head and eye movements were not recorded.

The American Association of State Highway and Transportation Officials (AASHTO, 2004) recommends "clear-sight triangles" to provide drivers with an unobstructed view of cross traffic at intersections. At a stop-controlled T-intersection, the sight triangle spans about $170^{\circ}$ (Figure 1). To view the whole area on the affected side, drivers with $\mathrm{HH}$ would have to make a gaze scan of about $85^{\circ}$ because they have no peripheral vision on that side (Figure 1). A gaze scan of this magnitude requires a large head movement (typically about $50^{\circ}$ (Bowers et al., 2014)) as well as an eye movement (e.g., about $35^{\circ}$ ). In a prior driving simulator study, intersection detection failures of drivers with $\mathrm{HH}$ were strongly associated with inadequate head scanning (Bowers et al., 2014). When drivers with left HH made a head scan toward the pedestrian on the far left in their blind hemifield, detection occurred only about $50 \%$ of the time and average head scan magnitudes were smaller when pedestrians were not detected than when they were detected. These results indirectly suggested that the gaze scan magnitude was inadequate; however, eye movements were not tracked so we were unable to determine how close the eyes (gaze) actually 
came to the pedestrian. Here we report a pilot study that addresses the relationship between gaze scanning and detection of pedestrians by drivers with $\mathrm{HH}$.

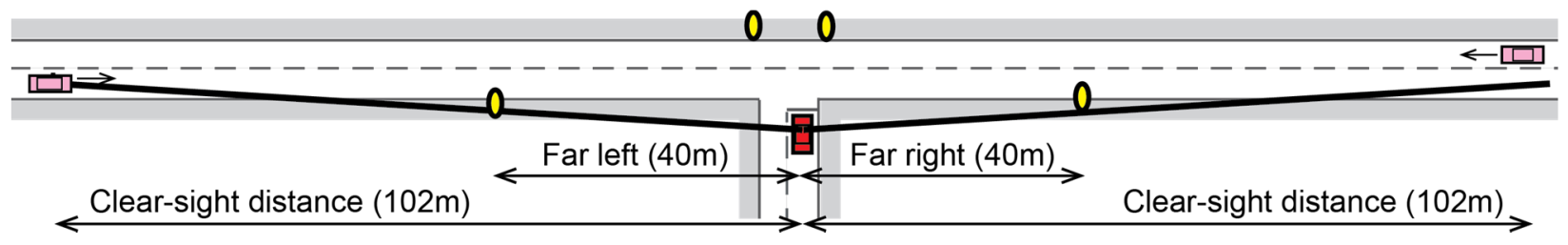

Figure 1: Schematic of the clear-sight triangle for a stop-controlled intersection at a $30 \mathrm{mph}$ cross street A driver with $\mathrm{HH}$ would have to execute a gaze scan of about $85^{\circ}$ to view the whole area of the sight triangle on the blind side. Pedestrians (yellow ovals) were placed at locations at the far left and far right of the sight triangle to evaluate whether drivers with HH scanned sufficiently far to the blind side. Pedestrians were also placed at center left and right to evaluate whether $\mathrm{HH}$ affected detection in more central parts of an intersection.

\section{METHODS}

Twelve people (mean age 39, range 18-82 years; $42 \%$ female) with complete $\mathrm{HH}$ (Goldmann V4e) and without spatial neglect (Bells test and Schenkenberg line bisection) participated. Stroke was the main cause $(67 \%)$ of the $\mathrm{HH}$. All had visual acuity of at least 20/40 and wore habitual spectacle corrections when driving; prism glasses were not used. The driving simulator (LE1500; FAAC Corp., Ann Arbor, MI) comprised five 42-in. LCD monitors providing a $225^{\circ}$ horizontal field of view. Gaze was tracked with a 6-camera remote eye-and-head tracking system (Smart Eye Pro; Goteborg, Sweden) which covered a range of at least $180^{\circ}$ enabling large magnitude gaze movements at intersections to be captured. Eye and head data were recorded at $60 \mathrm{~Hz}$ and synchronized with the simulator data stream, recorded at $30 \mathrm{~Hz}$.

Participants completed a series of test drives at each of two sessions including three routes on city roads (30 mph) with a variety of traffic situations and intersections. Audio cues (e.g., "turn left at next intersection") directed the driver along the routes. While driving they performed a pedestrian detection task (pressing the horn on detection). There were more than 100 pedestrian events across the two sessions, including 10 at intersections. Only pedestrians at intersections are considered in this paper; data for the other pedestrians were reported previously (Alberti et al., 2014). The pedestrian figure ( $2 \mathrm{~m}$ tall) was stationary and appeared at $10 \mathrm{~T}$-intersections with stop or yield signs across the two sessions: four times at the far left of the intersection (twice on a right turn and twice on a left turn) and twice at each of the other locations, center left (on a left turn only), center right (on a left turn only) and far right (on a right turn only) (Figure 1). None of the intersection pedestrians presented an imminent threat as they were stationary. Rather they were used to evaluate detection at locations where moving traffic could be a potential hazard for a turning vehicle. However, there was cross traffic on intersections with and without pedestrians. Based on the AASHTO clear-sight triangle for a stop-controlled intersection with a $30 \mathrm{mph}$ cross street, pedestrians were placed 40m along the sidewalk from the intersection to the left ("far left") and to the right ("far right") to evaluate whether drivers with left HH and right HH scanned sufficiently far $\left(85^{\circ}\right)$ to their blind sides, respectively. In addition, pedestrians were placed at center left and center right to evaluate whether $\mathrm{HH}$ affects detection in more central parts of an intersection critical to safe execution of a turn maneuver. Each pedestrian appeared as the driver was slowing to a stop at the intersection and disappeared as soon as the driver had completed a turn; only one pedestrian appeared at a time. 


\section{CATEGORIZING GAZE BEHAVIORS}

To examine the relationship between gaze movements and pedestrian detection at intersections, plots of lateral gaze position and pedestrian eccentricity were generated for each pedestrian event (Figures 2 and 3). Gaze movements at intersections typically comprised a series of large movements: large lateral head rotations with eye saccades. The movements took the eyes away from the straight ahead position to the left or right with a subsequent large movement in the opposite direction bringing the eyes back to the center, sometimes directly continuing with a large movement to the other side. We use the term "scan" to describe the complete gaze movement from the straight ahead position to the furthest lateral position. Gaze scans were analyzed between the time when the pedestrian appeared, and either the time of the horn press (when detected) or the time when it disappeared (when not detected). Each pedestrian event was characterized by whether detection occurred, and by whether there were any scans in the direction of the pedestrian, and, if there was a scan, how close gaze came to the pedestrian.
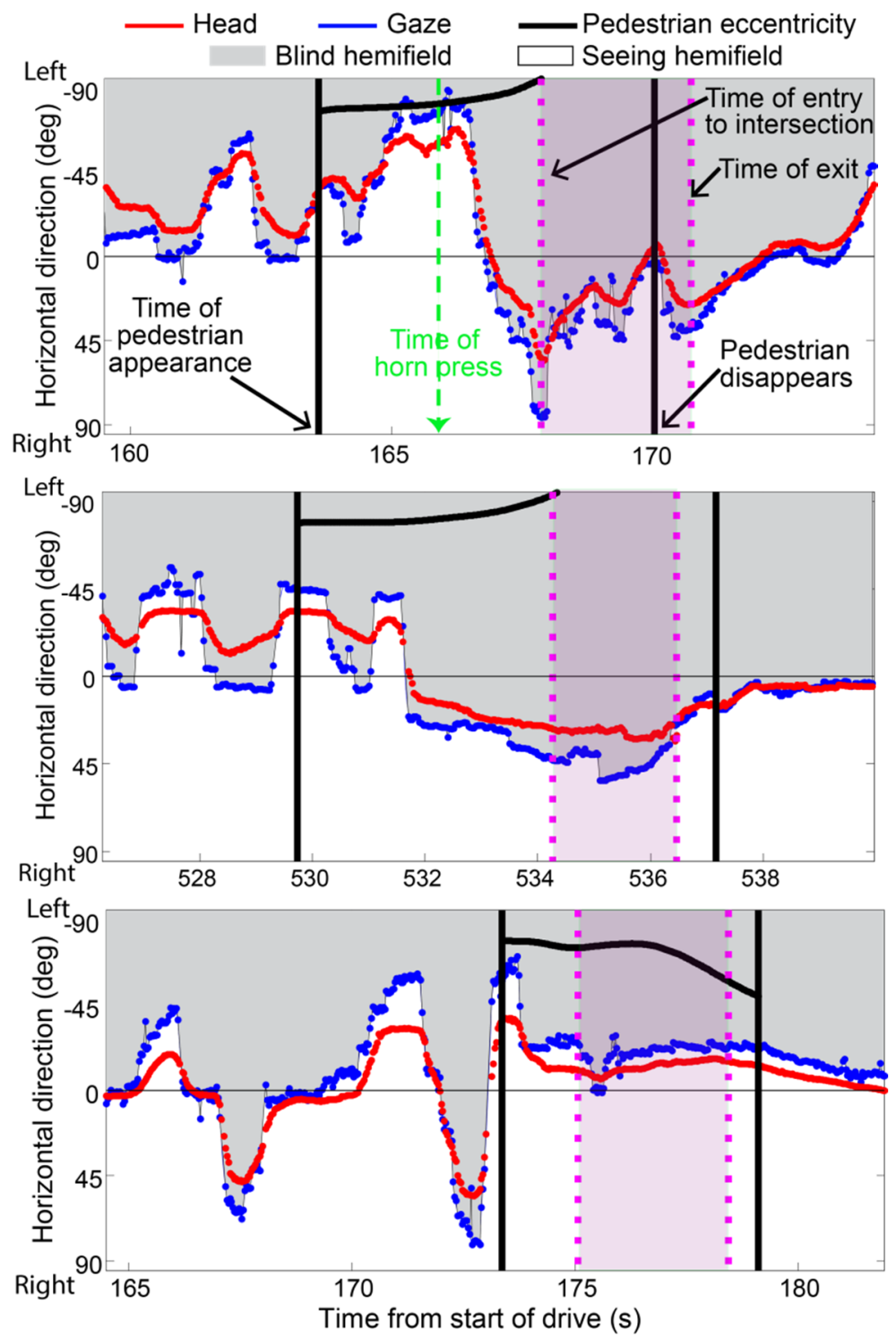

(a) Left $\mathrm{HH}$ driver detects pedestrian at far left (on the blind side) before a right turn. After the pedestrian appears, the driver makes a large leftward gaze scan $\left(\sim 83^{\circ}\right)$ which reaches the pedestrian in the blind hemifield at $165 \mathrm{~s}$. The pedestrian is then fixated. A horn press to indicate detection occurs toward the end of the fixation (about 166s). Pedestrian eccentricity exceeds $90^{\circ}$ after the car enters the intersection. (The difference between the gaze angle and head angle gives the eyein-head rotation.)

(b) Left $\mathrm{HH}$ driver fails to detect a pedestrian at far left (on the blind side) before a right turn due to inadequate gaze scan magnitudes. The leftward gaze scans of this driver are all about $45^{\circ}$, which is inadequate for detection of the pedestrian at $>80^{\circ}$. Pedestrian eccentricity exceeds $90^{\circ}$ after the car enters the intersection.

(c) Left HH driver fails to detect a pedestrian at far left (on the blind side) before a left turn due to inadequate gaze scan magnitude. The driver makes a large leftward gaze scan $\left(\sim 68^{\circ}\right)$ around $173 \mathrm{~s}$ which takes gaze close to the pedestrian at the time when it appears, but not close enough for detection to occur. (The pedestrian was at about $80^{\circ}$ eccentricity.)

Figure 2. Participants with left $\mathrm{HH}$ - plots of lateral head and gaze position 

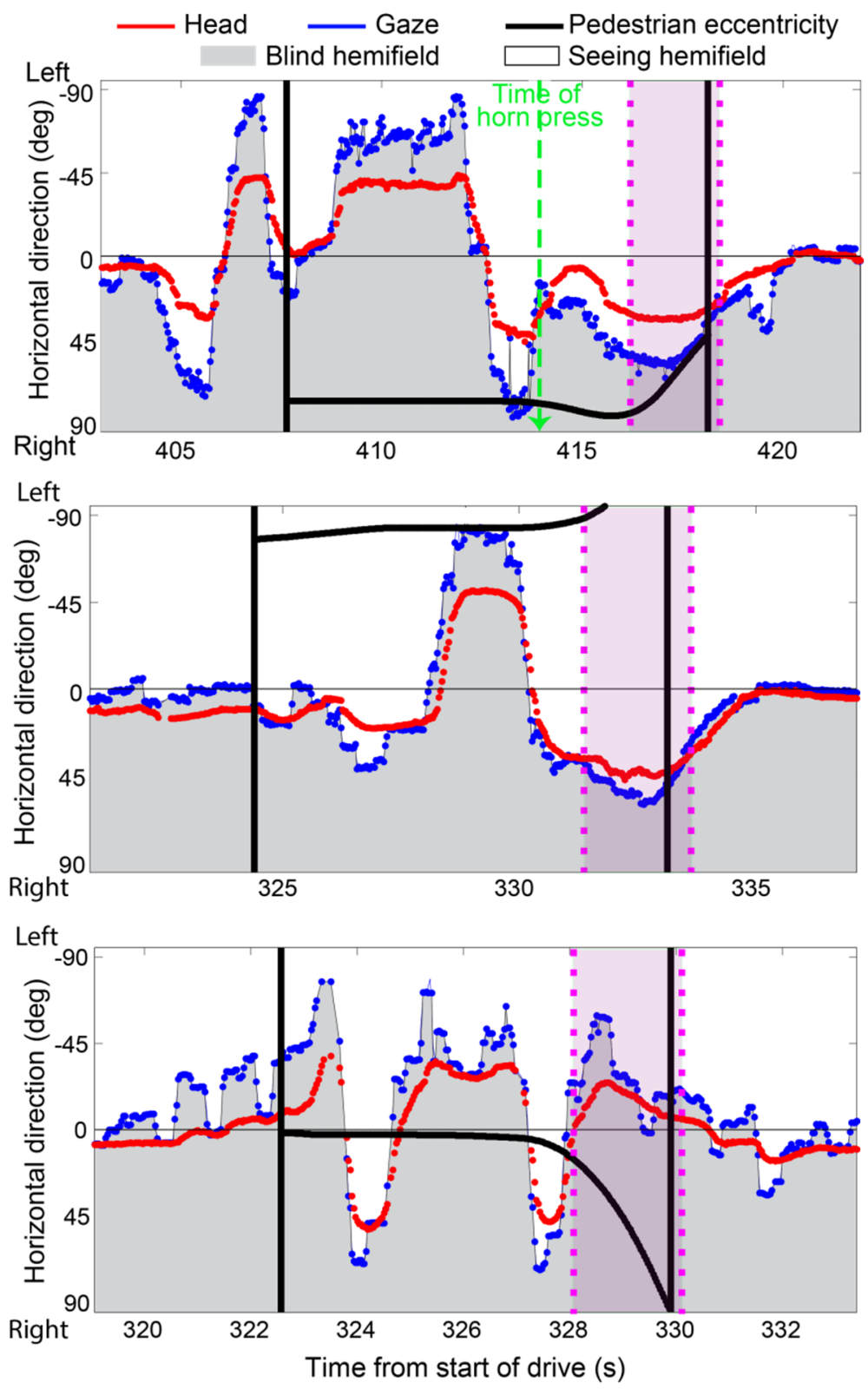

(a) Right $\mathrm{HH}$ driver detects a pedestrian at far right (on the blind side) before a right turn. The driver makes a large rightward gaze scan at about $413 \mathrm{~s}$ which takes gaze as far as the pedestrian, and the pedestrian is fixated. The horn press is shortly after the end of the fixation.

(b) Right $\mathrm{HH}$ driver fails to detect a pedestrian at far left (on the seeing side) before a left turn due to a looked-but-failedto-see (LBFTS) event. At about 328s, the driver makes a large leftward gaze scan toward the pedestrian, which finishes just to the right of the pedestrian. Although the pedestrian was in the seeing hemifield the whole time and gaze came very close, the pedestrian was not detected; therefore the event was categorized as LBFTS.

(c) Right HH driver fails to detect a pedestrian at center right before a left turn. The pedestrian appears in the blind right hemfield during a leftward gaze scan on the seeing side. Subsequent large gaze movements cross the pedestrian position in both directions, but the pedestrian is not detected; hence this was classifed as a "scan-across" event. Note that the pedestrian would be in the seeing hemifield whenever gaze is to the right of the pedestrian.

Figure 3. Participants with right $\mathrm{HH}$ - plots of lateral head and gaze position

Five main categories of gaze-detection behaviors were observed (Table 1). There were four categories for detection failures, two of which represented gaze scan deficits: no scan and inadequate gaze scan magnitude (e.g., Figures $2 \mathrm{~b}$ and c). The other two detection failure categories were looked-but-failed-to-see (LBFTS) (e.g., Figure 3b), which included situations where gaze scanning was adequate for detection to have occurred but there was no horn-press response, and scan-across (e.g, Figure 3c) which occurred for the center pedestrians only and was characterized by large gaze scans that took the eyes across the pedestrian in both directions such that it would have been in the seeing hemifield when a scan was toward the blind hemifield, but there was no horn-press response. The fifth category was seen (detected) for successful detections (Figures $2 \mathrm{a}$ and $3 \mathrm{a}$ ) 
Table 1: Five categories of gaze-detection behaviors

\begin{tabular}{lll}
\hline Detection & Gaze behavior & Description \\
\hline \multirow{2}{*}{ Not detected } & $\begin{array}{l}\text { No scan } \\
\text { Inadequate magnitude }\end{array}$ & $\begin{array}{l}\text { No gaze scan in the direction of the pedestrian } \\
\text { Gaze scan in the direction of the pedestrian but not far enough } \\
\end{array}$ \\
Scan across & $\begin{array}{l}\text { Pedestrian was fixated (or gaze came close in the seeing hemifield) } \\
\text { Large gaze scans took the eyes across the pedestrian in both } \\
\text { directions }\end{array}$ \\
\hline Detected & Fixated & $\begin{array}{l}\text { Fixated (or gaze came close enough for detection in seeing } \\
\text { hemifield) }\end{array}$ \\
\hline
\end{tabular}

\section{RESULTS}

The categorization of gaze-detection behaviors for each pedestrian location is summarized for drivers with left $\mathrm{HH}$ and right $\mathrm{HH}$ in Figures $4 \mathrm{a}$ and $4 \mathrm{~b}$, respectively.

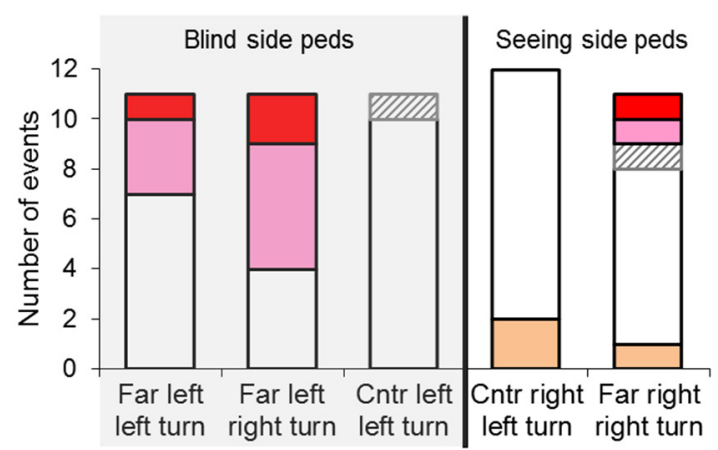

(a) Drivers with left $\mathrm{HH}$

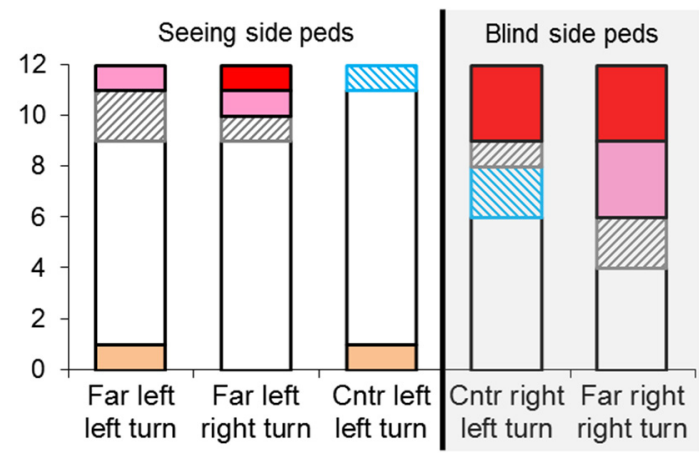

(b) Drivers with right $\mathrm{HH}$ $\square$ No scan

口Inadequate mag

口LBFTS

$₫$ Scan-across

口Seen

口Unclassified

Figure 4. Categorization of each pedestrian event for drivers

Data are presented as the number of events (pedestrian appearances) for data pooled across all participants in the left $\mathrm{HH}$ group and the right $\mathrm{HH}$ group. Total number of events is only 11 in some instances because two left HH drivers did not complete all the routes. Unclassified events are those with poor quality gaze tracking data.

Left pedestrians: Left $\mathrm{HH}$ and right $\mathrm{HH}$ drivers had similar detection rates (about $65 \%$ ) for the far-left pedestrian on a left turn, but left $\mathrm{HH}$ drivers had lower detection rates $(36 \%)$ than right $\mathrm{HH}$ drivers $(75 \%)$ on a right turn. Gaze scan deficits were the only reason for far-left detection failures by left $\mathrm{HH}$ drivers and were mainly inadequate gaze scan magnitudes before both a left and right turn. By comparison, far-left detection failures by right $\mathrm{HH}$ drivers included both gaze scan deficits and LBFTS incidents. Detection rates of both left and right $\mathrm{HH}$ drivers were high (over $90 \%$ ) for the center-left pedestrian. The only detection failure by a left HH driver was a single LBFTS incident. The only detection failure by a right $\mathrm{HH}$ driver was a single scan-across incident.

Right pedestrians: Right $\mathrm{HH}$ drivers had lower detection rates than left $\mathrm{HH}$ drivers for the center-right pedestrian (50\% vs. 92\%). Detection failures of right $\mathrm{HH}$ drivers included failing to gaze scan toward the pedestrian, a LBFTS incident and scan-across incidents. Right HH drivers also had lower detection rates than left $\mathrm{HH}$ drivers for the far-right pedestrian (33\% vs. 64\%). Far-right detection failures by left and right $\mathrm{HH}$ drivers included both gaze scan deficits and 
LBFTS incidents; however, scan deficits were the main reason for the detection failures by right HH drivers.

\section{DISCUSSION}

As expected, gaze scan deficits were the main reason for failing to detect pedestrians at the far locations at the edge of the clear-sight triangle in the blind hemifield. For drivers with left $\mathrm{HH}$, these deficits were more common on a right than a left turn, possibly suggesting a lower level of perceived threat from traffic from the left when making a right turn. Taken together, the results of this study and our prior simulator study (Bowers et al., 2014), suggest that drivers with HH frequently fail to head/gaze scan far enough to view the extreme areas of the clear-sight triangle on the blind side. Training to address these intersection scanning deficits may be beneficial, but has never been formally evaluated. At a minimum, drivers with $\mathrm{HH}$ should be advised about the need to fully scan the whole area of the intersection on their blind side. Specific instructions, e.g., to look into the blind hemifield until the sidewalk on the near side of the cross street is seen, may be helpful. Neither drivers with left $\mathrm{HH}$ nor right $\mathrm{HH}$ exhibited any gaze scan deficits for the center-left pedestrian which appeared before a left turn and came close to the car heading direction during the turn maneuver. By comparison, drivers with right $\mathrm{HH}$ did exhibit gaze scan deficits for the center-right pedestrian (on their blind side), which also appeared before a left turn. The eccentricity of this pedestrian increased with respect to the heading direction during the turn maneuver moving it further into the blind right hemifield, requiring either a small rightward gaze scan for detection before entering the intersection or a larger rightward gaze scan during the maneuver. The finding of gaze scan failures by drivers with right $\mathrm{HH}$ for the center-right pedestrian is consistent with the prior driving simulator study where drivers with right $\mathrm{HH}$ failed to head scan in the direction of that pedestrian. This gives cause for concern as the inability to detect an incoming car from the near right while making a left turn may result in a collision.

In addition to scanning deficits, the gaze data revealed that some of the detection failures had the characteristics of LBFTS incidents (Figure 3b), a form of inattentional blindness where an object is viewed without conscious awareness (Pammer \& Blink, 2013), which has been cited as a cause of intersection collisions (Koustanai et al., 2008). In this study, the horn-press response was the measure of awareness. If there was no response, the assumption was that the pedestrian had not been detected despite being fixated or gaze having come close enough for detection to occur. The gaze data also revealed an interesting "scan-across" phenomenon for the central pedestrians. In the example of the right HH driver shown in Figure 3c, the appearance of the pedestrian close to the center of the intersection was hidden by the hemifield loss because the driver was gazing to the far left at that time. Subsequent large gaze movements took the eyes across the pedestrian location, yet the pedestrian was not detected despite being in the seeing hemifield during scans toward the blind side. This situation appears to have the characteristics of a change blindness event, where a change in the scene is masked and subsequently goes undetected. Change blindness has been suggested as a mechanism underlying LBFTS events (White \& Caird, 2010). Although considered separately in our analysis, it seems that these scanacross events could have been categorized as LBFTS incidents.

In summary, we replicated the findings of our prior driving simulator study (Bowers, et al., 2014), and further confirmed that inadequate gaze scanning may increase the likelihood of a 
driver with $\mathrm{HH}$ failing to detect a potential hazard at an intersection. In addition, the gaze data revealed that some detection failures had the characteristics of LBFTS events. Drivers with a full field of vision were not included in this pilot; therefore, we could not evaluate whether the frequency of LBFTS events was higher for drivers with $\mathrm{HH}$ than normally-sighted drivers. The intersection pedestrians were stationary, did not present any threat to the driver, and appeared only infrequently (10 out of more than 100 pedestrian appearances in 2 hours of driving), which may have increased the likelihood of LBFTS events. A follow-up study with a larger sample size, more realistic intersection scenarios and control subjects is needed to evaluate the extent to which gaze scanning deficits impair detection of realistic moving hazards and whether $\mathrm{HH}$ increases the incidence of LBFTS events.

\section{ACKNOWLEDGEMENTS}

NIH grants EY018680, EY12890 and 1S10RR028122-01, and the Harvard Milton Fund.

\section{REFERENCES}

Alberti, C. F., Peli, E., \& Bowers, A. R. (2014). Driving with hemianopia: III. Detection of stationary and approaching pedestrians in a simulator. Investigative Ophthalmology and Visual Science, 55, 368-374.

American Association of State Highway and Transportation Officials. (2004). A Policy on Geometric Design of Highways and Streets. Washington, D.C.: AASHTO.

Bowers, A. R., Ananyev, E., Mandel, A. J., Goldstein, R. B., \& Peli, E. (2014). Driving with hemianopia: IV. Head scanning and detection at intersections in a simulator. Investigative Ophthalmology and Visual Science, 55, 1540-1548.

Bowers, A. R., Tant, M., \& Peli, E. (2012). A pilot evaluation of on-road detection performance by drivers with hemianopia using oblique peripheral prisms Stroke Research and Treatment, 2012( ), 176806

Koustanai, A., Boloix, E., Van Elslande, P., \& Bastien, C. (2008). Statistical analysis of "lookedbut-failed-to-see" accidents: Highlighting the involvement of two distinct mechanisms. Accident Analysis and Prevention, 40(2), 461-469.

Pammer, K., \& Blink, C. (2013). Attentional differences in driving judgments for country and city scenes: Semantic congruency in inattentional blindness. Accident Analysis and Prevention, 50, 955-963.

Papageorgiou, E., Hardiess, G., Mallot, H. A., \& Schiefer, U. (2012). Gaze patterns predicting successful collision avoidance in patients with homonymous visual field defects. Vision Research, 65, 25-37.

White, C. B., \& Caird, J. K. (2010). The blind date: The effects of change blindness, passenger conversation and gender on looked-but-failed-to-see (LBFTS) errors. Accident Analysis and Prevention, 42(6), 1822-1830.

Wood, J. M., McGwin, G., Elgin, J., Vaphiades, M. S., Braswell, R. A., DeCarlo, D. K., Kline, L. B., \& Owsley, C. (2011). Hemianopic and quadrantanopic field loss, eye and head movements, and driving. Investigative Ophthalmology \& Visual Science, 52(3), 1220-1225. 